\title{
Time Properties of the BuST Protocol under the NPA Budget Allocation Scheme
}

\author{
Gianluca Franchino \\ University of Pavia, Italy \\ gianluca.franchino@unipv.it
}

\author{
Giorgio C. Buttazzo \\ Scuola Superiore S. Anna, Italy \\ giorgio.buttazzo@sssup.it
}

\author{
Tullio Facchinetti \\ University of Pavia, Italy \\ tullio.facchinetti@unipv.it
}

\begin{abstract}
Token passing is a channel access technique used in several communication networks. Among them, one of the most effective solution for supporting both real-time traffic (synchronous messages) and non real-time traffic (asynchronous messages), is the so-called timed-token protocol. Recently, a new token passing protocol, called Budget Sharing Token protocol (BuST), was proposed to improve the existing timed-token approaches in terms of synchronous bandwidth guarantee, while guaranteeing a minimum throughput for the asynchronous traffic.

This paper analyzes the ability of BuST to manage realtime and non real-time traffic in comparison with the classic timed-token protocol and its modified version, under the Normalized Proportional Allocation (NPA) scheme. We will show that BuST achieves higher guaranteed realtime bandwidth than the original timed-token protocol, and improves the service for the non real-time traffic respect to its modified version.
\end{abstract}

\section{Introduction}

Many real-time control applications are nowadays distributed over several nodes having computational and communication capabilities. In such distributed systems, nodes collaborate, exchanging information, to achieve the application goal. Since nodes must complete their computational and control tasks within predefined time bounds (deadlines), they require the support of a real-time communication protocol to timely exchange information and thus to guarantee the correct behavior of the system, meeting task deadlines. Examples of such systems can be found in avionics, car control, factory automation, and telecommunication systems.

Different protocols have been proposed to manage the real-time communication among distributed nodes. One of the most popular solutions is represented by the timedtoken protocol, which was proposed for the first time in the early 1980s by Grow [8]. Since then, a lot of research has been done on this protocol (see [15]). The interest on studying token passing protocols arises from some features that make them interesting for various applications. For instance, the token passing mechanism allows the nodes to implicitly reclaim the bandwidth not used by the other nodes without having to add dedicated mechanisms to the protocol. Moreover, clock synchronization among the nodes is not required to properly run the protocol. Conversely, token passing networks can experience a high overhead when the token is lost and it has to be regenerated. An other possible shortcoming of this technique is the high jitter in message delivery.

Because of their features, timed-token protocols have been adopted by several communication standards as Medium Access Control (MAC) protocol. Examples of timed-token networks are PROFIBUS [14], Survivable Adaptable Fiber Optic Embedded Network (SAFENET) [10] and Fiber Distributed Data Interface (FDDI) [12]. In this paper we consider the timed-token protocol used in the MAC level of the FDDI standard and its modification named Modified FDDI (FDDI-M) [13].

In real-time communication systems, messages can be grouped in two classes: synchronous and asynchronous. The former class is primarily used for real-time messages with periodic arrival pattern, whereas the latter is used for non real-time aperiodic messages with unknown arrival time.

In timed-token approaches, an important parameter is the Target Token Rotation Time (TTRT), which represents the expected time needed by the token to complete an entire round of the network. Each node $i$ has an associated time budget $H_{i}$; whenever a node receives the token, it can transmit its synchronous messages for a time no greater than $H_{i}$. It can then transmit its asynchronous messages if the time elapsed since the previous token departure from the same node is less than the value of $T T R T$, that is, only if the token arrives earlier than expected. To assign the budget $H_{i}$ to each node, several allocation rules have been proposed during the past years. These rules are named Synchronous Budget Allocation (SBA) schemes.

BuST [7] is a token passing protocol recently introduced to improve the communication service provided by classic token protocols employed in the FDDI and FDDI$\mathrm{M}$ networks. The BuST protocol differs from FDDI and FDDI-M in how each node exploits the bandwidth saved during the round trip of the token, if any, to deliver asynchronous traffic. The transmission of asynchronous traffic occurs within the spare budget unused by synchronous traffic, even when the token is not early. In other words, 
the budget of a node is shared between real-time and non real-time traffic.

For evaluating and comparing the performance of different SBA schemes in a timed-token network, several metrics have been proposed. One of the most widely adopted is the Worst Case Achievable Utilization (WCAU) [9, 16]. The WCAU of an SBA scheme represents the largest utilization $\left(U^{*}\right)$ of the network such that, for any real-time message set whose total network utilization is $U \leq U^{*}$, the SBA scheme can guarantee the timeliness of each single real-time message. For a formal definition of the network utilization $U$ see Section 2.

The evaluation of WCAU is useful to guarantee the feasibility of a real-time message set when only an estimation of the amount of real-time traffic is known (i.e., the maximum time required to send a message) without requiring a detailed characterization of each single real-time message.

\subsection{Contributions and summary}

In [7], the authors showed that, when using the Proportional Allocation (PA) scheme, BuST outperforms FDDI and FDDI-M in the service of real-time traffic. In order to extend this previous work, in this paper we analyze the performance of BuST in managing both real-time and non real-time traffic, using the Normalized Proportional Allocation (NPA) scheme to allocate the node budgets. We compare the performance of BuST with FDDI and FDDIM.

Theoretical results show that, when the NPA scheme is used, BuST provides an higher WCAU with respect to FDDI, while it has the same WCAU of FDDI-M. However it is shown that FDDI-M can not deliver non realtime traffic; conversely BuST can deliver both real-time and non real-time traffic with the considered budget allocation scheme.

Moreover, we introduce new tests to guarantee the message scheduling with FDDI, FDDI-M and BuST protocols under the NPA scheme. The proposed tests are not based on the WCAU, but they use the protocol and messages parameters, e.g the deadlines (or the periods) and the TTRT.

The rest of the paper is organized as follows: Section 2 present the communication model; in Section 3 we briefly describe the BuST protocol; Section 4 introduces the NPA scheme considered in the analysis, and it provides theoretical results, as the WCAUs, for the discussed protocols when the NPA scheme is used; Section 5 shows the simulation results and finally, Section 6 states our conclusion and the future work.

\section{Communication model}

The communication system is composed by a set of $n$ communicating nodes. Each node $i$ is associated with a synchronous message stream $S_{i}$, which is described by three parameters $\left(C_{i}, T_{i}, D_{i}\right)$, where:

- $C_{i}$ is the maximum amount of time required to transmit a message in the stream. This includes the time required to transmit both the payload data and the message headers.
- $T_{i}$ is the interarrival period between consecutive messages in stream $S_{i}$. If the first message of node $i$ is put in the transmission queue at time $t_{i, 1}$, then the $j$ th message in stream $S_{i}$ will arrive at node $i$ at time $t_{i, j}=t_{i, 1}+(j-1) T_{i}$, where $j \geq 1$.

- $D_{i}$ is the relative deadline associated with messages in stream $S_{i}$, that is, the maximum amount of time that can elapse between a message arrival and the completion of its transmission. Thus, the transmission of the $j$-th message in stream $S_{i}$ that arrives at $t_{i, j}$ must be completed not later than $d_{i}=t_{i, j}+D_{i}$, which is the message's absolute deadline.

Without loss of generality, we assume only one synchronous stream per node. In fact, as proved in [1], a timed-token network with more than one stream per node can be transformed into a logically equivalent network with one synchronous stream per (logical) node.

Notice that, in order to guarantee the deadlines of asynchronous real-time messages, if any, we can use the stream model described above and assign a dedicated budget for this kind of messages, as proposed in [11].

The channel utilization of each message in the stream $S_{i}$ is

$$
U_{i}=\frac{C_{i}}{\min \left(T_{i}, D_{i}\right)} .
$$

The total effective channel utilization, $U$, of a periodic message set is then

$$
U=\sum_{i=1}^{n} U_{i}
$$

which measures the total channel bandwidth required by the whole periodic message set.

The parameters described above are crucial for guaranteeing the timely delivery of periodic messages. Before discussing how to select the communication parameters, we introduce the following definitions:

Definition $1 \tau$ is the time needed to transmit the token between nodes, including the overhead introduced by the protocol.

Any choice of the communication parameters must satisfy the following two constraints:

Definition 2 (Protocol Constraint) The total bandwidth allocated to the nodes must be less than the available network bandwidth, that is,

$$
\frac{\sum_{i=1}^{n} H_{i}}{T T R T} \leq 1-\frac{\tau}{T T R T} .
$$

The Protocol Constraint is necessary to ensure a stable operation of the timed-token protocol.

Definition 3 (Deadline Constraint) If $s_{i, j}$ is the time at which the transmission of the $j$-th message in stream $S_{i}$ is completed, the deadline constraint requires that for $i=$ $1, \ldots, n$ and $j=1,2, \ldots$,

$$
s_{i, j} \leq t_{i, j}+D_{i}
$$

where $t_{i, j}$ is the message arrival time and $D_{i}$ is its relative deadline. 
The Deadline Constraint ensures that every periodic message is transmitted before its absolute deadline. Note that in the above inequality, while $t_{i, j}$ and $D_{i}$ are defined by the application, $s_{i, j}$ depends on the synchronous bandwidth allocation and on the TTRT value.

\section{BuST protocol overview}

This section presents a brief description of BuST. Due to space limitations, we do not present the details of FDDI and FDDI-M. More information can be found in [7, 6].

It is known that, the main drawback of FDDI is the worst-case token rotation time, which is bounded by $2 T T R T$. Because of this, the timed-token protocol can only guarantee up to one half of the total available bandwidth for the real-time traffic. Instead, as we will see in Section 4.2, FDDI-M cannot deliver non real-time traffic under the NPA scheme. For further details on FDDI and FDDI-M problems see [7, 6].

The goal of the BuST protocol is to improve the original timed-token rules to avoid the problems of the timedtoken approach, just introduced above.

In particular, the worst-case token rotation time is limited so that it can not exceed the TTRT, which improves FDDI and allows a node to deliver non real-time traffic in those cases where FDDI-M fails.

Like in the traditional timed-token policy, the BuST protocol assigns each node a time budget $H_{i}$ for transmitting its real-time traffic. When a node receives the token, it can transmit the associated real-time traffic for a time no greater than the corresponding budget. The main difference with respect to FDDI and FDDI-M concerns the non real-time message service. Using FDDI, when the token arrives early, the node can transmit asynchronous traffic for a time no greater than $T_{A}=T T R T-\tau-T_{L R T}$, where $T_{L R T}$ is the time spent in the last round-trip of the token. Using FDDI-M a node does the same but with $T_{A}=T T R T-\sum_{i=1}^{n} H_{i}-\tau$. In BuST, a node can deliver non real-time traffic each time it gets the token, early or not, using the spare budget left by real-time messages. If $H_{i}^{\text {cons }}$ is the budget consumed by node $i$ to deliver synchronous traffic, then it can send asynchronous traffic for a time no greater than $T_{A_{i}}=H_{i}-H_{i}^{\text {cons }}$, even if the token is not early. Observe that, FDDI and FDDI-M can deliver asynchronous traffic only when the token is early, that is, when $T_{L R T}<T T R T-\tau$.

In BuST, node $i$ can use its budget $H_{i}$ for delivering both real-time and non real-time messages. Therefore, the worst-case token rotation time can not exceed TTRT. With respect to FDDI, BuST improves (as FDDI-M) the bandwidth available for real-time messages. For more detail on BuST see [7, 6].

\section{Time properties of NPA scheme}

The real-time guarantee of the stream set highly depends on the SBA scheme adopted for the budget assignment given the stream set parameters. In this paper we consider the Normalize Proportional Allocation (NPA) scheme. Such scheme has been extensively studied for FDDI and FDDI-M protocols, thus the time properties derived for BuST can be compared with the results available in the literature. The budget $H_{i}$ is allocated to node $i$ using the following equation:

$$
H_{i}=\frac{U_{i}}{U}(T T R T-\tau)
$$

Lemmas 1, 2, 3 calculate the bound on the maximum transmission time for real-time messages while using, BuST, FDDI-M and FDDI, respectively. As explicitly indicated in each lemma, the results are valid for all possible SBA schemes. These lemmas have been proved in [7], and due to space limitations the proofs are not reported in the paper.

Lemma 1 Under the BuST protocol, for all $S B A$ schemes, if $T_{i} \geq T T R T, i=1, \ldots, n$, it holds

$$
\forall i, j: s_{i, j} \leq t_{i, j}+\left\lceil\frac{C_{i}}{H_{i}}\right\rceil\left(\sum_{r=1}^{n} H_{r}+\tau\right) .
$$

Lemma 2 Under the FDDI-M protocol, for all $S B A$ schemes, if $T_{i} \geq T T R T, i=1, \ldots, n$, it holds

$$
\forall i, j: s_{i, j} \leq t_{i, j}+\left\lceil\frac{C_{i}}{H_{i}}\right\rceil \operatorname{TTRT}+C_{i}-\left\lceil\frac{C_{i}}{H_{i}}\right\rceil H_{i} .
$$

Lemma 3 Under the FDDI protocol, for all $S B A$ schemes, if $T_{i} \geq 2 T T R T, i=1, \ldots, n$, it holds

$\forall i, j: s_{i, j} \leq t_{i, j}+\left(\left\lceil\frac{C_{i}}{H_{i}}\right\rceil+1\right) T T R T+C_{i}-\left\lceil\frac{C_{i}}{H_{i}}\right\rceil H_{i}$.

\subsection{Normalized Proportional Allocation Scheme}

The results covered by Lemmas 1, 2 and 3 allow to prove the properties for the NPA scheme applied to BuST. Moreover, they can be used to derive new properties for the same scheme under FDDI and FDDI-M. When not differently indicated, the following results refer to the BuST protocol.

Notice that, even though we assume $D_{i}=T_{i}$ for all streams, since $U_{i}=\frac{C_{i}}{\min \left(D_{i}, T_{i}\right)}$, the same results can be derived for the case where $D_{i}<T_{i}$ by simply substituting $D_{i}$ to $T_{i}$.

To make the treatment clearer, let $\beta_{i}=\frac{T_{i}}{T T R T}$ and $\alpha=$ $\frac{\tau}{T T R T}$. Parameter $\alpha$ represents the bandwidth loss due to the protocol overhead.

Theorem 1 provides the WCAU for the Normalized Proportional Allocation scheme.

Theorem 1 The WCAU factor of the NPA scheme (where $\left.H_{i}=\frac{U_{i}}{U}(T T R T-\tau)\right)$ is equal to $\frac{1-\alpha}{2}$.

Proof. The Protocol Constraint is satisfied for any TTRT and for any $U$ since:

$$
\begin{aligned}
& \frac{1}{T T R T} \sum_{i=1}^{n} H_{i}=\frac{1}{T T R T} \sum_{i=1}^{n} \frac{U_{i}}{U}(T T R T-\tau)= \\
& \frac{T T R T-\tau}{T T R T} \leq 1-\frac{\tau}{T T R T} .
\end{aligned}
$$


For the Deadline Constraint, from Lemma 1, it holds:

$$
\begin{gathered}
s_{i, j} \leq t_{i, j}+\left\lceil\frac{C_{i}}{H_{i}}\right\rceil\left(\sum_{r=1}^{n} H_{r}+\tau\right)= \\
t_{i, j}+\left\lceil\frac{U \beta_{i}}{1-\alpha}\right\rceil\left(\sum_{r=1}^{n}\left(\frac{U_{r}}{U}(T T R T-\tau)\right)+\tau\right) \leq t_{i, j}+T_{i}
\end{gathered}
$$

Hence, to satisfy the deadlines for every messages in the stream $S_{i}$, the following inequality must hold:

$$
\begin{aligned}
& \left\lceil\frac{U \beta_{i}}{1-\alpha}\right\rceil\left(\sum_{r=1}^{n}\left(\frac{U_{r}}{U}(T T R T-\tau)\right)+\tau\right) \leq T_{i} \\
& \left\lceil\frac{U \beta_{i}}{1-\alpha}\right\rceil T T R T \leq T_{i} \\
& \left\lceil\frac{U \beta_{i}}{1-\alpha}\right\rceil \leq \beta_{i} .
\end{aligned}
$$

Being $\beta_{i} \geq 1$ and $\alpha \geq 0$, then $\frac{\beta_{i}}{1-\alpha} \geq 1$, hence it is not difficult to show (see [6]) that, if $U \leq \frac{1-\alpha}{2}$, the Deadline Constraint is met. $\square$

In FDDI-M [4], the WCAU is the same as in BuST, whereas for FDDI [5] is equal to $\frac{1-\alpha}{3}$.

Corollary 1 shows that, by properly setting TTRT, WCAU can be equal to the total available bandwidth for BuST and for FDDI-M. Instead, for FDDI, the WCAU can be equal to the half of the total available bandwidth. This latter is the maximum bandwidth that can be exploited to deliver real-time traffic under FDDI [7].

Corollary 1 If TTRT $=G C D_{i}\left(T_{i}\right)$, under BuST and $F D D I-M$, the WCAU factor of the NPA scheme is equal to the total available bandwidth, i.e., $1-\alpha$. Under FDDI, if TTRT $=0.5 \cdot G C D_{i}\left(T_{i}\right)$, the WCAU factor is equal to $\frac{1-\alpha}{2}$.

Proof. Notice that, the proof of Theorem 1 is valid to derive the WCAU both for BuST and for FDDI-M. Therefore, from the proof of Theorem 1, the Deadline Constraint is met under BuST and FDDI-M, if and only if:

$$
\left\lceil\frac{U \beta_{i}}{1-\alpha}\right\rceil \leq \beta_{i} .
$$

It is easy to see that, being $\beta_{i}=\frac{T_{i}}{G C D_{i}\left(T_{i}\right)} \in \mathbb{N}$, the deadline constraint is met if and only if $U \leq 1-\alpha$.

For FDDI, from Lemma 3, the Deadline Constraint is met if and only if

$$
\begin{aligned}
& \left(\left\lceil\frac{U \beta_{i}}{1-\alpha}\right\rceil+1\right) \operatorname{TTRT} \leq T_{i} \\
& \left\lceil\frac{U \beta_{i}}{1-\alpha}\right\rceil \leq \beta_{i}-1 .
\end{aligned}
$$

Being $\beta_{i}=\frac{T_{i}}{0.5 \cdot G C D_{i}\left(T_{i}\right)} \geq 2$ an integer, it is not difficult to verify that, if $U \leq \frac{1-\alpha}{2}$, then the deadline constraint is met. $\square$

Setting TTRT like in Corollary 1, being in the worst case $G C D_{i}\left(T_{i}\right)=1$, the bound for the utilization can get worse with respect to WCAU provided by Theorem 1 . This happens because $\alpha=\frac{\tau}{T T R T}$ can be very large.
Notice that, if TTRT $=G C D_{i}\left(T_{i}\right)$ then TTRT $\geq$ 1. Therefore, by setting TTRT as stated in Corollary 1 , substituting TTRT $=1$ in the schedulability condition $U \leq 1-\alpha$, it results that this last becomes $U \leq 1-\tau$, that is, the WCAU is equal to $1-\tau$ for BuST and FDDI$\mathrm{M}$. In the same way we can see that, by setting TTRT= $0.5 \cdot G C D_{i}\left(T_{i}\right)$, the WCAU becomes equal to $\frac{1}{2}-2 \tau$ for FDDI.

Theorem 2 provides a lower bound for the periods $T_{i}$ that guarantee the schedulability. Given the stream set, its utilization $U$ and the TTRT, this result can be used to check the schedulability. In particular, the following test can be exploited when $U>W C A U$.

Theorem 2 Consider a stream set $M=\left\{S_{1}, \ldots, S_{n}\right\}$, with utilization $U<1-\alpha$. Given TTRT $\leq T_{i}$, if $\forall i$ : $T_{i} \geq \operatorname{TTRT}\left(\left\lceil\frac{1}{1-\frac{U}{1-\alpha}}\right\rceil-1\right)$ or $\frac{T_{i}}{T T R T} \in \mathbb{N}$ then, under BuST and FDDI-M protocols with the NPA scheme, $M$ is schedulable.

Proof. Due to lack of space the proof is omitted. The interested reader can find the proof in [6].

A similar relationship between $T_{i}, T T R T$ and $U$, useful to test the schedulability for the FDDI protocol, is provided by the next theorem.

Theorem 3 Consider a stream set $M=\left\{S_{1}, \ldots, S_{n}\right\}$ with utilization $U<\frac{1-\alpha}{2}$. Given TTRT $\leq \frac{T_{i}}{2}$, if $\forall i: T_{i} \geq \operatorname{TTRT}\left(\left\lceil\frac{2}{1-\frac{U}{1-\alpha}}\right\rceil-1\right)$ or $\frac{T_{i}}{T T R T} \in \mathbb{N}$, then, under the FDDI protocol with the NPA scheme, $M$ is schedulable.

Proof. For the proof see [6].

\subsection{Non real-time service}

So far, real-time stream services have been analyzed. This subsection briefly describes the non real-time service of BuST and its improvements with respect to FDDI-M.

To analyze a worst-case scenario for the non real-time service, we assume that each node has always non realtime traffic to deliver when it receives the token. In this case, the total channel utilization of the network, including the real-time and the non real-time traffic, is equal to $1-\alpha$. Theorem 4 provides the minimum bandwidth that a node $i$ can exploit to deliver non real-time traffic.

Theorem 4 Under the BuST protocol, a node $i$ can guarantee for the non real-time traffic a minimum bandwidth $U_{i}^{n r t}$, which depends on the SBA scheme adopted. When the NPA scheme is used, it holds

$$
U_{i}^{n r t}=U_{i}\left(\frac{1-\alpha}{U}-1\right)
$$

Proof. The proof can be found in [6].

Theorem 4 shows that, using BuST, the non real-time traffic at each node has a minimum bandwidth guaranteed. Moreover, when some nodes have no non real-time traffic to send during the round trip of the token, the value of $U_{i}^{n r t}$ can increase. 
In the follow we show that, under the NPA scheme, FDDI-M can not deliver any non real-time traffic. Recalling that, with FDDI-M (see Section 3), the maximum time a node can use to deliver non real-time traffic is $T_{A}=T T R T-\sum_{i=1}^{n} H_{i}-\tau$, it follows that, when the NPA scheme is used:

$$
\begin{aligned}
T_{A}=\operatorname{TTRT}-\sum_{i=1}^{n} \frac{U_{i}}{U}(T T R T-\tau)-\tau & = \\
\operatorname{TTRT}-(T T R T-\tau)-\tau & =0
\end{aligned}
$$

In conclusion, BuST overcomes the impossibility of FDDI-M to deliver non real-time traffic with the NPA scheme, while maintaining the same WCAU of FDDI-M for the real-time traffic.

\subsection{Discussion of theoretic results}

Under the NPA scheme, BuST and FDDI-M have the same WCAU equal to $\frac{1-\alpha}{2}$. However, under this scheme FDDI-M can not deliver non real-time traffic. Conversely, as proved in Theorem 4, BuST can deliver also non realtime messages.

For FDDI under the NPA scheme we have a WCAU equal to $\frac{1-\alpha}{3}$, therefore BuST has a better WCAU respect to the timed-token protocol.

Corollary 1 state that, for NPA, the WCAU for BuST, FDDI-M, and FDDI can be improved by properly setting TTRT. In particular, the WCAU can get close to $1(0.5$ for FDDI) when $\alpha$ is negligible. However, TTRT may become very small, so that $\alpha=\frac{\tau}{T T R T}$ becomes big, resulting in a small available bandwidth, which also depend on the value of $\tau$.

For BuST and FDDI-M, Theorem 2 provides a simple test, base on the channel utilization $U, T T R T$ and message periods $T_{i}$, to verify the schedulability of a given stream set when the test on the WCAU fails. Theorem 3 gives a similar test for FDDI.

To show the usage of these new tests, consider a total channel utilization $U=0.8$, suppose to have TTRT $=2$ $m s$ and $\tau=0.2 \mathrm{~ms}$. Let $\gamma=\frac{U}{1-\alpha}=\frac{0.8}{1-(0.2 / 2)}$. By Theorem 2, any stream set with maximum channel utilization less than or equal to 0.8 , and with $\min _{i}\left(T_{i}\right) \geq$ $2\left(\left\lceil\frac{1}{1-\gamma}\right\rceil-1\right)=16 \mathrm{~ms}$ is schedulable with BuST and FDDI-M. Note that in this case we can not guarantee the schedulability by the WCAU, which is $\frac{1-\alpha}{2}=0.45$.

With FDDI, consider a channel utilization $U=0.4$ with the other parameters set as in the previous example. Note that, by Theorem 3, under FDDI $U$ must be less than $\frac{1-\alpha}{2}=0.45$. Let $\gamma=\frac{U}{1-\alpha}=\frac{0.4}{1-(0.2 / 2)}$, it follows that any stream set having $U \leq 0.4$, and $\min _{i}\left(T_{i}\right) \geq$ $2\left(\left\lceil\frac{2}{1-\gamma}\right\rceil-1\right)=6 \mathrm{~ms}$ is schedulable. Note that the proposed test is able to guarantee some stream set that the test based on the WCAU would miss, being $\frac{1-\alpha}{3}=0.3$.

\section{Simulation results}

In this section the performance of BuST, FDDI and FDDI-M is compared by simulation. The reported results refer to a network consisting of 10 nodes. Each node has a periodic stream with a relative deadline ranging from
$10 \mathrm{msec}$ to $100 \mathrm{msec}$, and an infinite amount of non realtime traffic, i.e. every time a node receives the token it has some non real-time traffic to deliver. It is worth noticing that in this case the total channel utilization, given by the sum of the real-time utilization $U$ and the non real-time utilization $U^{n r t}$, is equal to the total available bandwidth $1-\alpha$. Considering a protocol overhead for each node equal to $2 \mu \mathrm{sec}$, it follows that $\tau=20 \mu \mathrm{sec}$. We assigned the node budgets using the Normalized Proportional Allocation scheme analyzed in the previous sections. We measured the Maximum Deadline Miss Ratio varying the total channel utilization $U$ from 0.1 to 1.0 , with a step of 0.1 . For each value of $U$ we simulated the protocols with 500 runs, taking the Maximum value of the Deadline Miss Ratio. For each run we have generated a different stream set. In particular, for each stream set we have generated the utilizations $U_{i}$ randomly with a uniform distribution using the method proposed in [3]. For each value of $U_{i}$ we have generated a relative deadline $D_{i}$ randomly with a uniform distribution in the interval $[10 \mathrm{msec}, 100 \mathrm{msec}]$. Then the message lengths $C_{i}$ have been computed as $C_{i}=U_{i} D_{i}$. Notice that in the simulations we assume $D_{i}=P_{i}$.

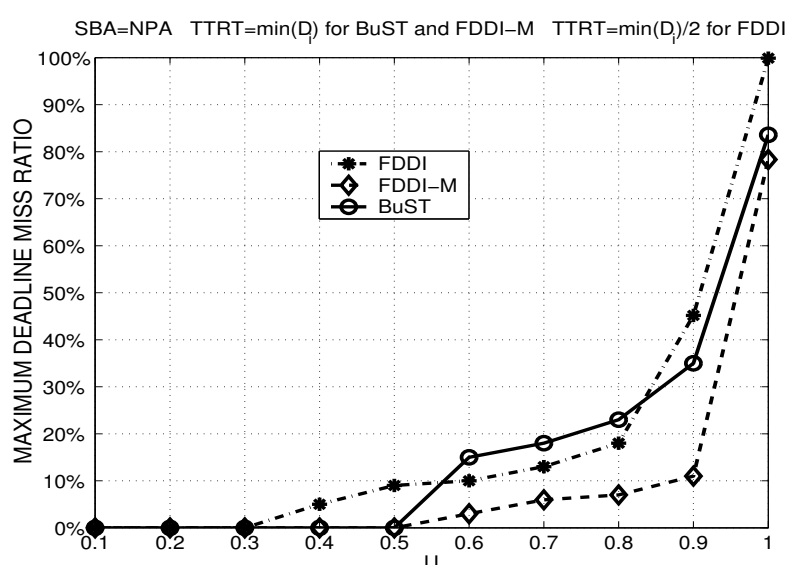

Figure 1. Maximum Deadline Miss Ratio for the NPA scheme.

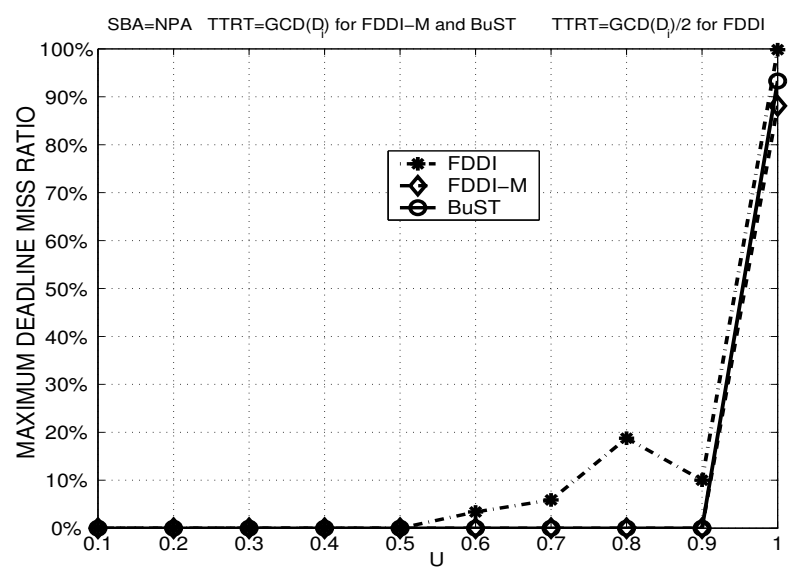

Figure 2. Maximum Deadline Miss Ratio with $T T R T=G C D_{i}\left(D_{i}\right)$.

Figure 1 reports the Maximum Deadline Miss Ratio. 
As expected from the analysis carried out in Section 4.1, as long as $U \leq \frac{1-\alpha}{2} \simeq 0.5$, BuST and FDDI-M have no deadline miss; for $U>0.5$ they start experiencing deadline misses.

For FDDI we have deadline misses for values of $U$ greater than 0.3 , as predicted by its WCAU which is $\frac{1-\alpha}{3} \simeq 0.33$. Notice that, simulating $U$ in the interval $[0.1,1]$ with steps of 0.1 , we do not simulate FDDI with $U=0.33$, but only with $U=0.3$ or $U=0.4$.

For $U>0.5$, the best performance is got by FDDI$\mathrm{M}$ and FDDI outperform BuST as long as $U \leq 0.8$. For $U>0.8$, BuST presents a better performance than FDDI. However, it is worth noticing again that FDDI-M is not delivering non real-time traffic, and this is the reason why it can provide a better service for real-time traffic. Conversely, BuST and FDDI can guarantee also the service for non real-time messages.

Figure 2 shows the results obtained when TTRT is set as stated in Corollary 1. In this case, as long as $U \leq$ 0.9 , both BuST and FDDI-M do not experience deadline misses. In fact, being $\alpha$ very small, the value of $U^{*}$ for both BuST and FDDI-M is close to 1 (see Corollary 1). FDDI does not have deadline miss for $U \leq 5$, as predicted by the theory.

We also simulated the behavior of the protocols when there is only real-time traffic in the network, that is, when the nodes has no non real-time traffic to deliver. In this case, FDDI, FDDI-M and BuST operate in the same way, since they are in practice the same protocol. In this case, in fact, the maximum token rotation time does not exceed TTRT even for FDDI. Therefore, TTRT can be set to $\min _{i}\left(D_{i}\right)$ for the three protocols. The performance of protocols are identical and represented by the dashed curve with diamonds points in Figure 1.

\section{Conclusions}

This paper analyzes the performance of the BuST protocol in comparison with FDDI and FDDI-M, when the NPA budget allocation scheme is used. Theoretical results show that BuST has equal or better performance with respect to the previous approaches. In particular, we showed that BuST has an higher WCAU than FDDI, and the same WCAU of FDDI-M. Moreover we showed that, with the NPA scheme, FDDI-M can not service asynchronous traffic, while BuST (as FDDI) can service also the asynchronous traffic.

Furthermore, we showed how to set the value of the TTRT to improve the bandwidth guaranteed for real-time streams under the NPA scheme for all the three considered protocols. We simulated the discussed protocols to confirm the theoretic results, and to evaluate their behavior under different conditions.

As a future work, we plan to compare BuST with other token passing protocols as, for instance, TDMA/SS [2]. We also plan to extend the analysis to other SBA schemes proposed in the literature for timed-token protocols and further investigate token loss problems related to the schedulability of real-time streams.

\section{References}

[1] G. Agrawal, B. Chen, W. Zhao, and S. Davari. Guaranteeing Synchronous Message Deadlines with the Timed Token Medium Access Control Protocol. IEEE Trans. on Computer, 43(3):327-339, March 1994.

[2] B. Andersson, E. Tovar, and N. Pereira. Analysing TDMA with Slot Skipping. In Proceedings of the 26th IEEE RealTime Systems Symposium, Dec. 2005.

[3] E. Bini and G. Buttazzo. Biasing effects in schedulability measures. In Proc. of the 16th IEEE Euromicro Conference on Real-Time Systems (ECRTS 2004), Jul. 2004.

[4] E. Chan, D. Chen, J. Cao, and C.-H. Lee. Time Properties of the FFDI-M Medium Access Protocol. The Computer Journal, 82(1):96-102, Jan. 1997.

[5] B. Chen and W. Zhao. Properties of the Timed Token Protocol, 92-038. Technical report, Dept. of Computer Science, Texas A\&M University College Station, Oct. 1992.

[6] G. Franchino, G. C. Buttazzo, and T. Facchinetti. A New Token Passing Protocol for Real-Time Communication. Technical report, University of Pavia, http://robot.unipv.it/media/publications/UNIPVRoboLab-Franchino-TR01-07.pdf, 2007.

[7] G. Franchino, G. C. Buttazzo, and T. Facchinetti. BuST: Budget Sharing Protocol for Hard Real-Time Communication. In Proc. of the 12th IEEE International Conference on Emerging Technologies and Factory Automation (ETFA 2007), Sept. 2007.

[8] R. Grow. A Timed Token Protocol for Local Area Networks. In Proc. Electro'82, Token Access Protocols, Paper 17/3, May 1982.

[9] M. Hamdaoui and P. Ramanathan. Selection of Timed Token Parameters to Guarantee Message Deadlines. IEEE/ACM Trans.on Networking, 3(3):340-351, 1995.

[10] R. J. Kochanski and J. L. Paige. SAFENET: The Standard and Its Application. IEEE LCS, 2(1):46-51, 1991.

[11] N. Malcolm and W. Zhao. The timed-token protocol for real-time communications. Computer, 27(1):35-41, Jan. 1994.

[12] K. Sevcik and M. Johnson. Cycle Time Properties of the FDDI Token Ring Protocol. IEEE Trans. on Software Eng., 13(3):376-385, March 1987.

[13] K. G. Shin and Q. Zheng. FDDI-M: A Scheme to Double FDDI's Ability of Supporting Synchronous Traffic. IEEE Trans. on Parallel and Distrib. Syst., 6(11):1125-1131, Nov. 1995.

[14] E. Tovar and F. Vasques. Cycle time properties of the PROFIBUS timed-token protocol. Computer Communications, Elsevier Science, 22(13):1206-1216, 1999.

[15] S. Zhang, A. Burns, J. Chen, and E. Lee. Hard Real-Time Communication with the Timed Token Protocol: Current State and Challenging Problems. Real-Time Systems, 27(3):271-295, Sept. 2004.

[16] S. Zhang, A. Burns, and A. Wellings. An Efficient and Practical Local Synchronous Bandwidth Allocation Scheme for The Timed-Token MAC Protocol. In Proc. of the IEEE Infocom'96, pages 920-927, 1996. 